\title{
Superintegrable Anharmonic Oscillators on $N$-dimensional Curved Spaces
}

\author{
Ángel Ballesteros $^{a}$, Alberto Enciso $^{b}$, Francisco José Herranz $^{c}$ and Orlando Ragnisco ${ }^{d}$ \\ ${ }^{a}$ Depto. de Física, Facultad de Ciencias, Universidad de Burgos, 09001 Burgos, Spain \\ E-mail: angelb@ubu.es
}

${ }^{b}$ Depto. de Física Teórica II, Universidad Complutense, 28040 Madrid, Spain

E-mail: aenciso@fis.ucm.es
${ }^{c}$ Depto. de Física, Escuela Politécnica Superior, Universidad de Burgos, 09001 Burgos, Spain E-mail: fjherranz@ubu.es

${ }^{d}$ Dipartimento di Fisica, Università di Roma Tre and Instituto Nazionale di Fisica Nucleare sezione di Roma Tre, Via Vasca Navale 84, 00146 Roma, Italy

E-mail: ragnisco@fis.uniroma3.it

\begin{abstract}
The maximal superintegrability of the intrinsic harmonic oscillator potential on $N$-dimensional spaces with constant curvature is revisited from the point of view of $s l$ (2)-Poisson coalgebra symmetry. It is shown how this algebraic approach leads to a straightforward definition of a new large family of quasi-maximally superintegrable perturbations of the intrinsic oscillator on such spaces. Moreover, the generalization of this construction to those $N$-dimensional spaces with non-constant curvature that are endowed with $s l(2)$-coalgebra symmetry is presented. As the first examples of the latter class of systems, both the oscillator potential on an $N$-dimensional Darboux space as well as several families of its quasi-maximally superintegrable anharmonic perturbations are explicitly constructed.
\end{abstract}

\section{Introduction}

The Poisson-coalgebraic "dynamical" symmetry underlying all the superintegrable Hamiltonian systems that we shall present in the sequel can be summarized as the following quite general result $[1,2]$ : Let $(\mathbf{q}, \mathbf{p})=\left(\left(q_{1}, \ldots, q_{N}\right),\left(p_{1}, \ldots, p_{N}\right)\right)$ be $N$ pairs of canonical variables with respect to the Poisson bracket

$$
\{f, g\}=\sum_{i=1}^{N}\left(\frac{\partial f}{\partial q_{i}} \frac{\partial g}{\partial p_{i}}-\frac{\partial g}{\partial q_{i}} \frac{\partial f}{\partial p_{i}}\right)
$$

and let us consider the three functions given by

$$
\mathbf{q}^{2}=\sum_{i=1}^{N} q_{i}^{2}, \quad \mathbf{p}^{2}=\sum_{i=1}^{N} p_{i}^{2}, \quad \mathbf{q} \cdot \mathbf{p}=\sum_{i=1}^{N} q_{i} p_{i} .
$$


Then, given any smooth function $\mathscr{H}$, the Hamiltonian

$$
H^{(N)}=\mathscr{H}\left(\mathbf{q}^{2}, \mathbf{p}^{2}, \mathbf{q} \cdot \mathbf{p}\right)
$$

defines an $N$-dimensional $(N D)$ classical superintegrable Hamiltonian $H^{(N)}$ with $(2 N-3)$ functionally independent integrals of the motion that are explicitly given by

$$
C^{(m)}=\sum_{1 \leq i<j}^{m}\left(q_{i} p_{j}-q_{j} p_{i}\right)^{2}, \quad C_{(m)}=\sum_{N-m+1 \leq i<j}^{N}\left(q_{i} p_{j}-q_{j} p_{i}\right)^{2},
$$

where $m=2, \ldots, N$ and $C^{(N)}=C_{(N)}$. Furthermore, the sets of functions given by $\left\{H^{(N)}, C^{(m)}\right\}$ and $\left\{H^{(N)}, C_{(m)}\right\}(m=2, \ldots, N)$ define two sets of $N$ integrals in involution. Proofs, technical details and further generalizations can be found in $[1,2]$ but, at this point, some remarks concerning the symmetry and superintegrability properties of $H^{(N)}$ are in order.

- Remark 1. If the three functions (1.2) are written as $J_{-}, J_{+}$and $J_{3}$, respectively, by computing the Poisson bracket (1.1) among them we recover the Lie-Poisson commutation rules of $\mathfrak{s l}(2, \mathbb{R})$ :

$$
\left\{J_{3}, J_{+}\right\}=2 J_{+}, \quad\left\{J_{3}, J_{-}\right\}=-2 J_{-}, \quad\left\{J_{-}, J_{+}\right\}=4 J_{3} .
$$

In other words, the functions (1.2) define a particular $N D$ symplectic realization of $\mathfrak{s l}(2, \mathbb{R})$. Hence any Hamiltonian $H^{(N)}$ can be thought of as a smooth function defined on $\mathfrak{s l}(2, \mathbb{R})$

$$
H^{(N)}=\mathscr{H}\left(J_{-}, J_{+}, J_{3}\right)=\mathscr{H}\left(\mathbf{q}^{2}, \mathbf{p}^{2}, \mathbf{q} \cdot \mathbf{p}\right),
$$

and all the results here presented can be interpreted in the framework of $\mathfrak{s l}(2, \mathbb{R})$-Poisson dynamics. Properly speaking, $H^{(N)}$ is defined on a three-dimensional $\mathfrak{s l}(2, \mathbb{R})$-subalgebra of the $\mathfrak{s l}(2, \mathbb{R}) \otimes \cdots \otimes^{N)} \mathfrak{s l}(2, \mathbb{R})$ Poisson algebra. We stress that integrable systems on the Euclidean space and endowed with the $N$-particle $\mathfrak{s l}(2, \mathbb{R})$-symmetry given by the representation (1.2), were already studied in [3].

- Remark 2. The "universal" integrals of motion (1.4) are derived from the Casimir function of the aforementioned $\mathfrak{s l}(2, \mathbb{R})$ Poisson algebra (see [1]), and are given as sums of the square of certain angular momentum components. In particular, since the functions $L_{i j}=q_{i} p_{j}-$ $q_{j} p_{i}$ with $i<j$ and $i, j=1, \ldots, N$ span an $\mathfrak{s o}(N)$ Lie-Poisson algebra with Poisson brackets

$$
\left\{L_{i j}, L_{i k}\right\}=L_{j k}, \quad\left\{L_{i j}, L_{j k}\right\}=-L_{i k}, \quad\left\{L_{i k}, L_{j k}\right\}=L_{i j}, \quad i<j<k,
$$

the integrals (1.4) can be rewritten as

$$
C^{(m)}=\sum_{1 \leq i<j}^{m} L_{i j}^{2}, \quad C_{(m)}=\sum_{N-m+1 \leq i<j}^{N} L_{i j}^{2} .
$$

This, in turn, means that the $\mathfrak{s l}(2, \mathbb{R}) \otimes \cdots \otimes \otimes^{N)} \mathfrak{s l}(2, \mathbb{R})$ symmetry gives us the right prescription to get the appropriate subset of quadratic functions of the generators of $\mathfrak{s o}(N)$ that Poisson-commute with the Hamiltonian (1.3) and are in involution. In this respect, note that the algebra $\mathfrak{s l}(2, \mathbb{R}) \otimes \cdots \otimes^{N)} \mathfrak{s l}(2, \mathbb{R})$ has only $3 N$ generators and many of them do Poisson-commute, whilst $\mathfrak{s o}(N)$ has $N(N-1) / 2$ generators with many non-vanishing Poisson brackets among them. 
- Remark 3. It is well-known that the maximum number of functionally independent (and different from the Hamiltonian itself) integrals of the motion for an ND Hamiltonian is $(2 N-2)$. In the case that all these integrals do exist, the sytem is called maximally superintegrable (MS). Since $H^{(N)}(1.3)$ has, by construction, $(2 N-3)$ functionally independent integrals, we shall say that this is a quasi-maximally superintegrable (QMS) Hamiltonian. Nevertheless, for some specific choices of the function $\mathscr{H}$ it will be possible to find the remaining integral (which is not provided by the above symmetry). In that case $\mathscr{H}$ will define a MS system.

- Remark 4. The canonical variables $(\mathbf{q}, \mathbf{p})$ have a priori neither a given geometrical (physical) meaning, nor restricted (real/complex) values.

Therefore, we can conclude that $H^{(N)}$ (1.6) comprises a large family of QMS Hamiltonians; each particular system arises for a specific choice of the function $\mathscr{H}$ together with an "appropriate" geometrical interpretation of the canonical variables.

\subsection{Oscillators on the $N D$ Euclidean space}

In order to illustrate these ideas and also as the starting point for further developments, let us consider the $N \mathrm{D}$ isotropic harmonic oscillator with angular frequency $\omega$. Such a system can easily be identified within the family (1.6) by simply setting

$$
\mathscr{H}=\frac{1}{2} J_{+}+\frac{1}{2} \omega^{2} J_{-}=\frac{1}{2} \mathbf{p}^{2}+\frac{1}{2} \omega^{2} \mathbf{q}^{2}
$$

for which $\mathbf{q}$ are Cartesian coordinates in the $N D$ Euclidean space $\mathbb{E}^{N}$. This Hamiltonian is not only QMS, but the standard prototype of MS systems. In fact, the "remaining" constant of the motion can be taken as any of the $N$ integrals

$$
\mathscr{I}_{i}=p_{i}^{2}+\omega^{2} q_{i}^{2}, \quad i=1, \ldots, N,
$$

since each $\mathscr{I}_{i}$ is functionally independent with respect to both the set (1.4) and $\mathscr{H}$. The results above summarized allows for a straightforward superintegrable even-order anharmonic oscillator perturbation given by [1]:

$$
\mathscr{H}=\frac{1}{2} J_{+}+\frac{1}{2} \omega^{2} J_{-}+\sum_{k=1}^{\infty} \delta_{k} J_{-}^{k+1}=\frac{1}{2} \mathbf{p}^{2}+\frac{1}{2} \omega^{2} \mathbf{q}^{2}+\sum_{k=1}^{\infty} \delta_{k} \mathbf{q}^{2(k+1)},
$$

which is QMS for any choice of the $\delta_{k}$ parameters, since the Hamiltonian (1.11) is indeed a function of (1.2). We remark that once a single anharmonic contribution with parameter $\delta_{k}$ is added to the first harmonic term the maximal superintegrability is lost, but the resulting system (for any number of arbitrary $\delta_{k}$ 's) always keeps the $(2 N-3)$ integrals of motion (1.4). In particular, the latter are just the integrals of the motion for the radial Garnier system $[4,5]$, which is recovered by taking $\omega$ and $\delta_{1}$ as the only non-vanishing parameters. We also recall that the integrability properties of some quartic oscillators can be generalized to the Calogero-Moser systems defined with such nonlinear oscillators as external potentials (see [6] and references therein).

In the following sections we present the superintegrable Hamiltonians defining anharmonic oscillators on the $N \mathrm{D}$ sphere and hyperbolic spaces [7], as well as on an ND Riemannian space 
of variable curvature. The latter space is an ND generalization of the 2D Darboux surface of type III $[8,9]$, one of the four 2D spaces with non-constant curvature whose geodesic flows are MS. We stress that in the constant curvature cases the zero-curvature (flat) limit leads to the Euclidean nonlinear oscillator (1.11).

\section{Anharmonic oscillators on spaces of constant curvature}

In this section we consider the $N D$ classical Riemannian spaces with constant sectional curvature $\kappa$ : the sphere $\mathbb{S}^{N}(\kappa>0)$ and the hyperbolic space $\mathbb{H}^{N}(\kappa<0)$. We recall that both of them can be embedded in a linear space $\mathbb{R}^{N+1}$ with ambient or Weierstrass coordinates $\left(x_{0}, \mathbf{x}\right)=\left(x_{0}, x_{1}, \ldots, x_{N}\right)$ subjected to the "sphere" constraint

$$
\Sigma: \quad x_{0}^{2}+\kappa \mathbf{x}^{2}=1 .
$$

The metric on the proper $N D$ spaces reads [10]:

$$
\mathrm{d} s^{2}=\left.\frac{1}{\kappa}\left(\mathrm{d} x_{0}^{2}+\kappa \mathrm{d} \mathbf{x}^{2}\right)\right|_{\Sigma}
$$

where $\mathrm{d} \mathbf{x}^{2}=\sum_{i=1}^{N} \mathrm{~d} x_{i}^{2}$.

In order to be able to apply the results described in section 1 to the geodesic flows and oscillator potentials on $\mathbb{S}^{N}$ and $\mathbb{H}^{N}$ (with the construction of the constant curvature counterpart of the QMS Hamiltonian on $\mathbb{E}^{N}$ (1.11) in mind), we shall proceed as follows:

- We interpret in a proper way the "abstract" canonical coordinates and momenta $(\mathbf{q}, \mathbf{p})$ as intrinsic quantities on each space; this step can be achieved through different projections from the ambient space $\mathbb{R}^{N+1}$. The resulting metric in terms of $\mathbf{q}$ leads to the kinetic energy term of the Hamiltonian.

- We deduce the form of the corresponding intrinsic harmonic oscillator potential on the spaces with constant curvature as a function of $\mathbf{q}$; its flat limit (or contraction) $\kappa \rightarrow 0$ has to give (1.9). Moreover, in our framework the well-known maximal superintegrability of this potential on these spaces has to be explicitly proven by finding the additional integral of the motion through direct computation.

- Finally, QMS anharmonic oscillator potentials can be obtained as a symmetry-preserving perturbation of the intrinsic oscillator on these spaces: in particular, we can consider the sum of all the powers of the above intrinsic oscillator potential in such a way that their flat limit $\kappa \rightarrow 0$ reduces to (1.11). In this way, the constant curvature analogues of the radial Garnier system will be obtained by considering the anharmonicity given by the square of the intrinsic oscillator.

In the following we apply the above steps by considering two types of phase spaces $(\mathbf{q}, \mathbf{p})$ in $\mathbb{S}^{N}$ and $\mathbb{H}^{N}$ coming from different projections from $\mathbb{R}^{N+1}$. Obviously, the two Hamiltonians so obtained are canonically equivalent through a change of coordinates despite its apparent disequivalence as objects defined on the $\mathfrak{s l}(2, \mathbb{R})$ Poisson algebra. 


\subsection{Stereographic projection: Poincaré coordinates}

Let us consider the stereographic projection [7] from the ambient coordinates $\left(x_{0}, \mathbf{x}\right) \in \Sigma \subset \mathbb{R}^{N+1}$ to the Poincaré coordinates $\mathbf{q} \in \mathbb{R}^{N}$ with pole $(-1, \mathbf{0}) \in \mathbb{R}^{N+1}$ :

$$
(-1, \mathbf{0})+\lambda(1, \mathbf{q}) \in \Sigma .
$$

Hence we obtain that

$$
\lambda=\frac{2}{1+\kappa \mathbf{q}^{2}}, \quad x_{0}=\lambda-1=\frac{1-\kappa \mathbf{q}^{2}}{1+\kappa \mathbf{q}^{2}}, \quad \mathbf{x}=\lambda \mathbf{q}=\frac{2 \mathbf{q}}{1+\kappa \mathbf{q}^{2}} .
$$

Therefore, the metric (2.2) in Poincaré coordinates reads

$$
\mathrm{d} s^{2}=4 \frac{\mathrm{d} \mathbf{q}^{2}}{\left(1+\kappa \mathbf{q}^{2}\right)^{2}} .
$$

And the associated geodesic flow has (up to a positive constant factor) the free Lagrangian

$$
\mathscr{T}=\frac{\dot{\mathbf{q}}^{2}}{2\left(1+\kappa \mathbf{q}^{2}\right)^{2}}
$$

The canonical momenta $\mathbf{p}$ are obtained through the usual Legendre transformation and read:

$$
\mathbf{p}=\frac{\dot{\mathbf{q}}}{\left(1+\kappa \mathbf{q}^{2}\right)^{2}}
$$

Thus the $N D$ kinetic energy on $\mathbb{S}^{N}$ and $\mathbb{H}^{N}$ is given in Hamiltonian form as a particular case of (1.6); namely

$$
\mathscr{T}=\frac{1}{2}\left(1+\kappa J_{-}\right)^{2} J_{+}=\frac{1}{2}\left(1+\kappa \mathbf{q}^{2}\right)^{2} \mathbf{p}^{2} .
$$

As a consequence, this geodesic flow is (at least) QMS, with (1.4) being the explicit (and invariant) form of the integrals of the motion. However, we stress that in the context of Poincare coordinates the geometric interpretation of such integrals in terms of angular momentum components is lacking.

The next point is to deduce the curved harmonic oscillator potential in terms of these Poincare coordinates q. We recall that the radial (geodesic polar) distance $r$ from an arbitrary point to the origin in $\mathbb{S}^{N}(\kappa>0)$ and $\mathbb{H}^{N}(\kappa<0)$ along the geodesic joining both points is written (in ambient coordinates) as $[10,11]$

$$
\frac{1}{\kappa} \tan ^{2}(\sqrt{\kappa} r)=\frac{\mathbf{x}^{2}}{x_{0}^{2}}
$$

Consequently, the well-known intrinsic oscillator on constant curvature spaces (the so-called Higgs oscillator $[12,13])$ is written in Poincaré coordinates as

$$
\mathscr{U}=\frac{1}{2} \omega^{2} \frac{\mathbf{q}^{2}}{\left(1-\kappa \mathbf{q}^{2}\right)^{2}} .
$$


In this way the full Higgs oscillator Hamiltonian reads

$$
\mathscr{H}=\frac{1}{2}\left(1+\kappa J_{-}\right)^{2} J_{+}+\frac{1}{2} \omega^{2} \frac{J_{-}}{\left(1-\kappa J_{-}\right)^{2}}=\frac{1}{2}\left(1+\kappa \mathbf{q}^{2}\right)^{2} \mathbf{p}^{2}+\frac{1}{2} \omega^{2} \frac{\mathbf{q}^{2}}{\left(1-\kappa \mathbf{q}^{2}\right)^{2}} .
$$

This system is known to be MS [12]. Therefore, the remaining functionally independent constant of the motion does exist and, therefore, it has to be found by direct methods. Such an additional integral can be shown to be any of the following $N$ functions [1]:

$$
\mathscr{I}_{i}=\left(p_{i}\left(1-\kappa \mathbf{q}^{2}\right)+2 \kappa(\mathbf{q} \cdot \mathbf{p}) q_{i}\right)^{2}+\frac{\omega^{2} q_{i}^{2}}{\left(1-\kappa \mathbf{q}^{2}\right)^{2}}, \quad i=1, \ldots, N
$$

Now, a natural perturbation of this Hamiltonian including anharmonic terms that preserve the QMS properties of the system would be

$$
\begin{aligned}
\mathscr{H} & =\frac{1}{2}\left(1+\kappa J_{-}\right)^{2} J_{+}+\frac{1}{2} \omega^{2} \frac{J_{-}}{\left(1-\kappa J_{-}\right)^{2}}+\sum_{k=1}^{\infty} \delta_{k}\left(\frac{J_{-}}{\left(1-\kappa J_{-}\right)^{2}}\right)^{k+1} \\
& =\frac{1}{2}\left(1+\kappa \mathbf{q}^{2}\right)^{2} \mathbf{p}^{2}+\frac{1}{2} \omega^{2} \frac{\mathbf{q}^{2}}{\left(1-\kappa \mathbf{q}^{2}\right)^{2}}+\sum_{k=1}^{\infty} \delta_{k} \frac{\mathbf{q}^{2(k+1)}}{\left(1-\kappa \mathbf{q}^{2}\right)^{2(k+1)}} .
\end{aligned}
$$

Notice that when any $\delta_{k} \neq 0$ this curved anharmonic oscillator is QMS (it always commutes with the integrals (1.4) due to its $\mathfrak{s l}(2, \mathbb{R})$-coalgebra symmetry) but not MS (at least with integrals depending quadratically on the momenta). In fact, the $N=2$ restriction of this perturbed system does not appear in the classifications of MS systems on $\mathbb{S}^{2}$ and $\mathbb{H}^{2}$ given in $[14,15,16]$. Note also that the first perturbative term given by $\delta_{1} \neq 0$ can be considered as the constant curvature generalization of the (radial) Garnier system.

\subsection{Central projection: Beltrami coordinates}

Now we consider the central projection from the ambient coordinates $\left(x_{0}, \mathbf{x}\right) \in \Sigma \subset \mathbb{R}^{N+1}$ to the Beltrami ones $\mathbf{q} \in \mathbb{R}^{N}$ with pole $(0, \mathbf{0}) \in \mathbb{R}^{N+1}$ :

$$
(0, \mathbf{0})+\mu(1, \mathbf{q}) \in \Sigma
$$

so that we find

$$
\mu=\frac{1}{\sqrt{1+\kappa \mathbf{q}^{2}}}, \quad x_{0}=\mu, \quad \mathbf{x}=\mu \mathbf{q}=\frac{\mathbf{q}}{\sqrt{1+\kappa \mathbf{q}^{2}}} .
$$

Then the metric (2.2) turns out to be

$$
\mathrm{d} s^{2}=\frac{\left(1+\kappa \mathbf{q}^{2}\right) \mathrm{d} \mathbf{q}^{2}-\kappa(\mathbf{q} \cdot \mathrm{d} \mathbf{q})^{2}}{\left(1+\kappa \mathbf{q}^{2}\right)^{2}} .
$$

The corresponding free Lagrangian is given by

$$
\mathscr{T}=\frac{\left(1+\kappa \mathbf{q}^{2}\right) \dot{\mathbf{q}}^{2}-\kappa(\mathbf{q} \cdot \dot{\mathbf{q}})^{2}}{2\left(1+\kappa \mathbf{q}^{2}\right)^{2}}
$$


which leads to the definition of the conjugate momenta $\mathbf{p}$ as:

$$
\mathbf{p}=\frac{\left(1+\kappa \mathbf{q}^{2}\right) \dot{\mathbf{q}}-\kappa(\mathbf{q} \cdot \dot{\mathbf{q}}) \mathbf{q}}{\left(1+\kappa \mathbf{q}^{2}\right)^{2}}
$$

Hence the kinetic energy Hamiltonian describing geodesic motion reads

$$
\mathscr{T}=\frac{1}{2}\left(1+\kappa J_{-}\right)\left(J_{+}+\kappa J_{3}^{2}\right)=\frac{1}{2}\left(1+\kappa \mathbf{q}^{2}\right)\left(\mathbf{p}^{2}+\kappa(\mathbf{q} \cdot \mathbf{p})^{2}\right) .
$$

By taking into account (2.9) we find that the expression of the curved oscillator on $\mathbb{S}^{N}$ and $\mathbb{H}^{N}$ adopts in these Beltrami coordinates the following "Euclidean" form:

$$
\mathscr{U}=\frac{1}{2} \omega^{2} \mathbf{q}^{2},
$$

which yields the following expression for the complete curved oscillator Hamiltonian (again as a particular case of (1.6)):

$$
\mathscr{H}=\frac{1}{2}\left(1+\kappa J_{-}\right)\left(J_{+}+\kappa J_{3}^{2}\right)+\frac{1}{2} \omega^{2} J_{-}=\frac{1}{2}\left(1+\kappa \mathbf{q}^{2}\right)\left(\mathbf{p}^{2}+\kappa(\mathbf{q} \cdot \mathbf{p})^{2}\right)+\frac{1}{2} \omega^{2} \mathbf{q}^{2} .
$$

The remaining constant of the motion for this MS Hamiltonian can be taken from any of the $N$ functions [1]

$$
\mathscr{I}_{i}=\left(p_{i}+\kappa(\mathbf{q} \cdot \mathbf{p}) q_{i}\right)^{2}+\omega^{2} q_{i}^{2}, \quad i=1, \ldots, N .
$$

And the explicit QMS anharmonic generalization of (2.21) is proposed to be

$$
\begin{aligned}
\mathscr{H} & =\frac{1}{2}\left(1+\kappa J_{-}\right)\left(J_{+}+\kappa J_{3}^{2}\right)+\frac{1}{2} \omega^{2} J_{-}+\sum_{k=1}^{\infty} \delta_{k} J_{-}^{k+1} \\
& =\frac{1}{2}\left(1+\kappa \mathbf{q}^{2}\right)\left(\mathbf{p}^{2}+\kappa(\mathbf{q} \cdot \mathbf{p})^{2}\right)+\frac{1}{2} \omega^{2} \mathbf{q}^{2}+\sum_{k=1}^{\infty} \delta_{k} \mathbf{q}^{2(k+1)} .
\end{aligned}
$$

Note that in this coordinates the curved Garnier term is given just by the $\mathbf{q}^{4}$ perturbation.

\section{Oscillators on an ND space of non-constant curvature}

In arbitrary manifolds with non-constant curvature, kinetic-energy Hamiltonians can exhibit extremely complicated dynamics and are, in general, no longer integrable [17]. From a physical viewpoint, the caracterization of (super)integrable geodesic flows on curved (pseudo)-Riemannian manifolds in arbitrary dimensions is relevant for supergravity and superstring theories, and constitutes an active research field (see [18] and references therein). Let us consider the (spherically symmetric and conformally flat) ND Riemannian manifold whose metric and geodesic Hamiltonian flow are given by

$$
\mathrm{d} s^{2}=\left(a+\mathbf{q}^{2}\right) \mathrm{d} \mathbf{q}^{2}, \quad \mathscr{T}=\frac{\mathbf{p}^{2}}{a+\mathbf{q}^{2}},
$$


where the parameter $a>0$. This is a space with non-constant curvature. Moreover, its scalar curvature is negative and given by

$$
R=-(N-1) \frac{3(N-2) \mathbf{q}^{2}+2 a N}{\left(a+\mathbf{q}^{2}\right)^{3}} .
$$

In the $N=2$ case, this space is just one of the so-called 2D Darboux spaces: the 2-manifolds with non-constant curvature admitting two quadratic first integrals, so that its geodesic motion is quadratically MS. There are only four types of such spaces [8], which are (from the integrability viewpoint) the closest ones to constant curvature ones, since their $N \mathrm{D}$ generalizations are the only spaces other than $\mathbb{E}^{N}, \mathbb{H}^{N}$ and $\mathbb{S}^{N}$ whose geodesic motion could be expected to be (quadratically) MS for any dimension. In fact, an ND spherically symmetric generalization of the four 2D Darboux spaces has been recently introduced in [19] and shown to be QMS.

The integrability properties of the space (3.1) have been thoroughly studied in [20], where it has been shown that the Hamiltonian

$$
\mathscr{H}=\frac{J_{+}+\omega^{2} J_{-}}{a+J_{-}}=\frac{\mathbf{p}^{2}}{a+\mathbf{q}^{2}}+\omega^{2} \frac{\mathbf{q}^{2}}{a+\mathbf{q}^{2}},
$$

is a MS system with $(2 N-2)$ functionally independent quadratic first integrals given again by (1.4) and one of the following functions

$$
\mathscr{I}_{i}=p_{i}^{2}-\left(\mathscr{H}-\omega^{2}\right) q_{i}^{2}, \quad i=1, \ldots, N .
$$

Note that this integral cannot be written as a function of the $\mathfrak{s l}(2, \mathbb{R})$ symmetry; moreover, the set $\left\{\mathscr{I}_{i}: 1 \leq i \leq N\right\}$ is also in involution. To the best of our knowledge, this Hamiltonian provides the first example of a Hamiltonian system on a Riemannian space of non-constant curvature which is MS in any dimension.

Furthermore, a geometric analysis shows [20] that the potential

$$
\mathscr{U}=\omega^{2} \frac{J_{-}}{a+J_{-}}=\omega^{2} \frac{\mathbf{q}^{2}}{a+\mathbf{q}^{2}},
$$

can be interpreted as the intrinsic harmonic oscillator on this curved space, that turns out to be MS, despite of the introduction of a non-constant curvature. We remark that, as expected, for $N=2$ this model is listed in the classification of MS potentials for the Darboux space of type III given in [9]. At this point, two different proposals for the definition of an ND QMS anharmonic oscillator perturbation on this space arise in a natural way. The first one consists in the same type of generalization proposed in the constant curvature cases described in section 2:

$$
\begin{aligned}
\mathscr{H} & =\frac{J_{+}}{a+J_{-}}+\omega^{2} \frac{J_{-}}{a+J_{-}}+\sum_{k=1}^{\infty} \delta_{k}\left(\frac{J_{-}}{a+J_{-}}\right)^{k+1} \\
& =\frac{\mathbf{p}^{2}}{a+\mathbf{q}^{2}}+\omega^{2} \frac{\mathbf{q}^{2}}{a+\mathbf{q}^{2}}+\sum_{k=1}^{\infty} \delta_{k}\left(\frac{\mathbf{q}^{2}}{a+\mathbf{q}^{2}}\right)^{k+1} .
\end{aligned}
$$

A second (and perhaps more natural) possibility arises if we realize that in (3.3) the intrinsic oscillator on this curved space is just the "Euclidean" oscillator divided by the conformal factor of 
the metric, which carries all the information concerning the non-constant curvature of the space (see [19]). From this perspective, the curved anharmonic oscillator would be defined as

$$
\mathscr{H}=\frac{J_{+}+\omega^{2} J_{-}+\sum_{k=1}^{\infty} \delta_{k} J_{-}{ }^{k+1}}{a+J_{-}}=\frac{\mathbf{p}^{2}+\omega^{2} \mathbf{q}^{2}+\sum_{k=1}^{\infty} \delta_{k} \mathbf{q}^{2(k+1)}}{a+\mathbf{q}^{2}} .
$$

In any case, both Hamiltonians (3.6) and (3.7) are QMS and, like the rest of the systems presented in this paper, they do have the same set of universal integrals (1.4) coming from their $\mathfrak{s l}(2, \mathbb{R})$ symmetry.

Acknowledgments. This work was partially supported by the Spanish MEC and by the Junta de Castilla y León under grants no. FIS2004-07913, MTM2007-67389 and VA013C05 (A.B. and F.J.H.), by the Spanish DGI under grant no. FIS2005-00752 (A.E.) and by the INFN-CICyT (O.R.). A.E. acknowledges the financial support of the Spanish MEC through an FPU scholarship. A.B. and F.J.H. are also grateful to G.S. Pogosyan for helpful discussions.

\section{References}

[1] Ballesteros A and Herranz F J, Universal integrals for superintegrable systems on $N$ dimensional spaces of constant curvature, J. Phys. A: Math. Theor. 40 (2007), F51-F59.

[2] Ragnisco O, Ballesteros A, Herranz F J and Musso F, Quantum deformations and superintegrable motions on spaces with variable curvature, SIGMA 3 (2007), 026(20).

[3] Wojciechowski S, Construction of integrable systems by dressing a free motion with a potential, Phys. Lett. A 96 (1983), 389-392.

[4] GARniER R, Sur une classe de systèmes différentiels abéliens déduits de la théorie des équations linéaires, Rend. Circ. Math. Palermo 43 (1919), 155-191.

[5] Wojciechowski S, Integrability of one particle in a perturbed central quartic potential, Physica Scripta 31 (1985), 433-438.

[6] WojcIEchowski S, On the solvability of the Calogero-Moser system in some external potentials and of other related Hamiltonians systems, Phys. Lett. A 104 (1984), 189-192.

[7] Doubrovine B, Novikov S and Fomenko A, Géométrie Contemporaine, Méthodes et Applications, First Part, MIR, Moscow, 1982.

[8] Koenigs G, Sur les géodésiques à intégrales quadratiques, in: G. Darboux (Ed.), Leçons sur la théorie générale des surfaces, Vol. 4, Chelsea, New York, 1972, 368-404.

[9] Kalnins E G, Kress J M, Miller W and Winternitz P, Superintegrable systems in Darboux spaces, J. Math. Phys. 44 (2003), 5811-5848.

[10] Ballesteros A, Herranz F J, Santander M and Sanz-Gil T, Maximal superintegrability on N-dimensional curved spaces, J. Phys. A: Math. Gen. 36 (2003), L93-L99.

[11] Herranz F J, Ballesteros A, Santander M and Sanz-Gil T, Maximally superintegrable Smorodinsky-Winternitz systems on the $N$-dimensional sphere and hyperbolic spaces, in: P. Tempesta et al (Eds.), Superintegrability in Classical and Quantum Systems, CRM Proceedings and Lecture Notes 37, AMS, Providence, 2004, 75-89. 
[12] Higgs P W, Dynamical symmetries in a spherical geometry I, J. Phys. A: Math. Gen. 12 (1979), 309-323.

[13] Leemon H I, Dynamical symmetries in a spherical geometry II, J. Phys. A: Math. Gen. 12 (1979), 489-501.

[14] RAÑADA M F and SANTANDER M, Superintegrable systems on the two-dimensional sphere $S^{2}$ and the hyperbolic plane $H^{2}$, J. Math. Phys. 40 (1999), 5026-5057.

[15] Kalnins E G, Miller W and Pogosyan G S, Completeness of multiseparable superintegrability on the complex 2-sphere, J. Phys. A: Math. Gen. 33 (2000), 6791-6806.

[16] Kalnins E G, Kress J M, Pogosyan G S and Miller W, Completeness of superintegrability in two-dimensional constant-curvature spaces, J. Phys. A: Math. Gen. 34 (2001), 4705-4720.

[17] Paternain G P, Geodesic flows, Birkhäuser, Boston, 1999.

[18] Gibbons G W, LÜ H, Page D N and Pope C N, The general Kerr-de Sitter metrics in all dimensions, J. Geom. Phys. 53 (2005), 49-73.

[19] Ballesteros A, Enciso A, Herranz F J, Ragnisco O, $N$-dimensional sl(2)-coalgebra spaces with non-constant curvature, Phys. Lett. B 652 (2007), 376-383.

[20] Ballesteros A, Enciso A, Herranz F J, Ragnisco O, A maximally superintegrable system on an $n$-dimensional space of nonconstant curvature, Physica D 237 (2008), 505-509. 